\title{
Complexo de parasitóides de Phyllocnistis citrella (Lepidoptera, Gracillariidae) em dois pomares de citros em Montenegro, RS, Brasil
}

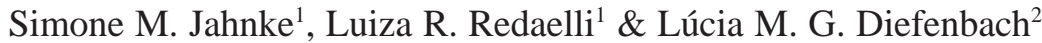

1. Departamento de Fitossanidade, Universidade Federal do Rio Grande do Sul, Av. Bento Gonçalves, 7712, 91540-000 Porto Alegre, RS, Brasil(smjahnke@yahoo.com; luredael@ufrgs.br)

2. Instituto de Pesquisas Biológicas, Laboratório Central de Saúde Pública do Estado do Rio Grande do Sul (IPB-LACEN/RS), Fundação Estadual de Produção e Pesquisa em Saúde (FEPPS), Av. Ipiranga, 5400, 90610-000 Porto Alegre, RS, Brasil (luciadiefenbach@aol.com)

\begin{abstract}
Phyllocnistis citrella (Lepidoptera, Gracillariidae) and its parasitoids in two citrus orchards in Montenegro, RS, Brazil. Several parasitoid wasps, mainly from Eulophidae and Chalcididae families, have been registered acting upon the citrus leafminer Phyllocnistis citrella Stainton, 1856 populations, one of the most important citrus pest worldwide. The present work aimed to identify the parasitoid complex of $P$. citrella in two citrus orchards, located in Montenegro, RS: one of the Montenegrina cultivar and the other of the hybrid tangor Murcott. In fortnightly samplings from July 2001 to June 2003, all the buds of randomly rafted plants were inspected. All the leaves, with pupae of P. citrella, were collected and individually maintained in Petri dishes until the emergence of parasitoids or adults of $P$. citrella. In the first year, 101 parasitoid individuals of the genus Elasmus Westwood, 1833, Cirrospilus Westwood, 1832, Sympiesis Förster, 1856 and Galeopsomyia fausta LaSalle, 1997 (Eulophidae) were obtained. Ageniaspis citricola Logvinovskaya, 1983 (Encyrtidae) was registered only in the 'Murcott' orchard. This species was intentionally introduced in nearby orchards for the leafminer control. In the second year, a total of 609 parasitoid individuals were sampled, and A. citricola was also registered in the 'Montenegrina' area. With the introduction and establishment of the exotic species in the orchards, occurred a great change in the species relative frequency. Ageniaspis citricola represented more than $75 \%$ of the individuals occurring in both orchards in the second year, and the relative frequency of the native species decreasing. Studies on the community of natural enemies associated to the citrus leafminer, and on the actual impact that the exotic species cause on the native ones are necessary before taking any measure of control.
\end{abstract}

KEYWORDS. Citrus leafminer, parasitoid, Hymenoptera, biological control, Eulophidae.

RESUMO. Diversas vespas parasitóides, especialmente Eulophidae e Chalcididae, têm sido registrados atuando sobre populações de Phyllocnistis citrella Stainton, 1856 (larva-minadora-dos-citros), uma das principais pragas da citricultura mundial. O presente trabalho objetivou identificar o complexo de parasitóides de $P$. citrella em dois pomares de citros situados em Montenegro, RS, um de tangerineira variedade Montenegrina e outro do híbrido tangor Murcott. Em amostragens quinzenais de julho de 2001 a junho de 2003 , todos os brotos de plantas sorteadas aleatoriamente eram inspecionados. Todas as folhas com pupas de $P$. citrella foram coletadas e acondicionadas individualmente em placas de Petri até a emergência dos parasitóides ou dos adultos de $P$. citrella. No primeiro ano foram obtidos 101 parasitóides, dos gêneros Elasmus Westwood, 1833, Cirrospilus Westwood, 1832, Sympiesis Förster, 1856 e Galeopsomyia fausta LaSalle, 1997 (Eulophidae). Ageniaspis citricola Logvinovskaya, 1983 (Encyrtidae) foi registrada somente na área de 'Murcott'. Esta espécie foi intencionalmente introduzida em pomares próximos para o controle do minador. No segundo ano foi amostrado um total de 609 parasitóides e A. citricola foi também registrada na área de 'Montenegrina'. Com a introdução e o estabelecimento da espécie exótica nos pomares, houve uma grande mudança na freqüência relativa das espécies. Ageniaspis citricola representou mais de $75 \%$ dos indivíduos presentes em ambos pomares no segundo ano, diminuindo a freqüência relativa das espécies nativas. Estudos sobre a comunidade de inimigos naturais associados ao minador-dos-citros, e o efetivo impacto que a espécie exótica causa sobre as nativas, são necessários antes de tomarem-se quaisquer medidas de controle.

PALAVRAS-CHAVE. Minador-das-folhas-dos-citros, parasitóide, Hymenoptera, controle biológico, Eulophidae.

O minador-das-folhas-dos-citros, Phyllocnistis citrella Stainton, 1856 (Lepidoptera; Gracillariidae), é um microlepidóptero originário do sudeste Asiático. No Brasil, sua presença foi registrada pela primeira vez em 1996 (Prates et al., 1996).

Devido à ação minadora das larvas nas folhas, $P$. citrella pode causar danos diretos e indiretos às plantas de citros. A redução da superfície fotossintética, o enrolamento das folhas criando um microambiente propício para o desenvolvimento de afídeos e cochonilhas e a queda prematura de folhas e brotações são alguns dos danos provocados (Generalitat Valenciana, 1996). Indiretamente, a larva minadora pode abrir passagem para a entrada da bactéria Xanthomonas citri pv. citri, causadora do cancro cítrico. Levantamentos realizados em vários países têm demonstrado um índice de infestação de $X$. citri pv. citri de até $75 \%$ em folhas de citros, quando P. citrella está presente (HePpNER, 1993; Willink et al., 1996; Chagas \& Parra, 2000). A combinação destes fatores faz com que $P$. citrella seja considerada uma praga importante e alvo de uma série de estudos nos países produtores de citros.

O minador, como a maioria dos insetos fitófagos, ao invadir os novos ecossistemas, provavelmente não veio acompanhado de seus inimigos naturais, o que contribuiu para que, rapidamente, alcançasse a condição de praga. Para Urbaneja et al. (1998), este processo de dispersão faz com que haja uma adaptação de parasitóides oportunistas autóctones, que ocupam nichos ecológicos similares, podendo, esses, tornarem-se fatores chaves na regulação das populações destes fitófagos.

Dentre os parasitóides autóctones registrados no 
Brasil e associados a $P$. citrella, os mais freqüentes são espécies de Eulophidae, Eupelmidae e Chalcididae (PEnTEAdo-Dias et al., 1997; SÁ et al., 1999; NASCIMENTO et al., 2000).

Gravena (1998) aponta Galeopsomyia fausta LaSalle, 1997 (Eulophidae) como a espécie de parasitóide mais importante, em São Paulo, pois aparece numa frequiência de $90 \%$ em relação a outras presentes. Também foram registradas em pomares de laranja de São Paulo e do Rio de Janeiro, diferentes espécies de Cirrospilus Westwood, 1832 e Elasmus Westwood, 1833 (SÁ et al., 2000, MonTES et al., 2001). Em Santa Catarina, GARCIA et al. (2001) constataram a presença dos eulofídeos Elasmus sp., Cirrospilus sp., G. fausta e uma espécie de Elachertus Spinola, 1811.

No Rio Grande do Sul, os levantamentos, embora preliminares, apontaram a presença de espécies de Elasmus, Cirrospilus, Aprostocetus Westwood, 1833, Horismenus Walker 1843 e G. fausta (Eulophidae) sem, entretanto, registrarem dados quantitativos a respeito das espécies ou índices de parasitismo (BECKER \& Moraes, 2001).

O conhecimento das espécies de parasitóides que atuam sobre populações de $P$. citrella, ao longo do tempo, é fundamental para o estabelecimento de manejos adequados, que busquem o controle da mesma. Neste sentido, o presente trabalho objetivou realizar o levantamento de espécies parasitóides de $P$. citrella presentes em dois pomares de citros no município de Montenegro, RS e registrar possíveis diferenças na composição e estrutura das assembléias.

\section{MATERIAL E MÉTODOS}

O trabalho foi realizado, durante dois anos, de julho de 2001 a junho de 2003, no município de Montenegro $\left(29^{\circ} 68^{\prime} \mathrm{S}, 51^{\circ} 46^{\prime} \mathrm{W}\right)$ em dois pomares contíguos, de tangerineiras da variedade Montenegrina (Citrus deliciosa Tenore) e do híbrido tangor Murcott ( $C$. sinensis $\mathrm{L}$. Obseck $\mathrm{x} C$. reticulata Blanco), com aproximadamente 2 ha cada um. O manejo das áreas não inclui a aplicação de herbicidas, nem a liberação de agentes biológicos de controle.

No primeiro ano, quinzenalmente, de julho de 2001 a junho de 2002, a cada ocasião de amostragem eram sorteadas aleatoriamente 12 plantas (aproximadamente 4\% das árvores) de cada área, de onde foram retiradas as unidades de amostra. No segundo ano de amostragem, de julho de 2002 a junho de 2003, buscando aumentar o número de unidades de amostras, optou-se por monitorar 24 plantas de cada área.

Em cada planta sorteada foram inspecionados todos os brotos para verificar a presença de câmaras pupais de $P$. citrella. Todas as folhas contendo estas câmaras foram colhidas e acondicionadas em sacos plásticos etiquetados, contendo algodão embebido em água, para manter a turgidez das mesmas. Em laboratório, as folhas foram individualizadas em placas de Petri, vedadas e mantidas, sob condições ambientais, até a emergência dos parasitóides ou dos adultos de $P$. citrella.

A identificação dos parasitóides foi realizada através de chave dicotômica (PENTEADO-Dias et al., 1997) até o nível de família e enviadas ao Instituto Biológico de Campinas para a identificação específica.

Determinou-se a freqüência relativa de cada espécie, com relação ao total de indivíduos em cada um dos pomares estudados (CALLEGARI-JACQUES, 2004). A constância, que avalia a porcentagem de espécies presentes nos levantamentos efetuados, foi calculada segundo DAJOZ (1973).

\section{RESULTADOS E DISCUSSÃO}

Um total de 52 ocasiões de amostragem, em cada pomar, resultou na captura de 710 parasitóides emergidos de pupas de $P$. citrella, pertencentes a Hymenoptera sendo seis espécies de Eulophidae e uma de Encyrtidae.

Os representantes de Eulophidae compreenderam Cirrospilus neotropicus (Diez \& Fidalgo, 2003), Cirrospilus floridensis Evans, 1999 (Cirrospilini), este último registrado pela primeira vez no Brasil por JAHNKE et al. (2005), Sympiesis sp. (Eulophini), G. fausta (Tetrastichini) e Elasmus sp. 1 e sp. 2 (Elasmini). As espécies de Sympiesis e Elasmus, ainda não foram descritas e nomeadas, constando apenas como morfotipos (Schauff et al., 1998). A família Eulophidae inclui muitas espécies de parasitóides de importantes pragas de plantas cultivadas (SCHAUFF et al., 1998; BURKS, 2001). Cirrospilus neotropicus foi a mais frequiente, quando comparada às outras espécies nativas presentes nas áreas. Em todo Brasil, também já foram apontadas várias espécies de Eulophidae, dos gêneros Cirrospilus, Elasmus, Pediobus e Sympiesis e G. fausta, parasitando P. citrella (Penteado-Dias et al., 1997; NAscimento et al., 2000).

Detectou-se ainda, neste estudo, a presença de Ageniaspis citricola Logvinovskaya, 1983 (Encyrtidae), espécie exótica que havia sido introduzida em agosto de 2001 em várias propriedades em áreas próximas aos pomares estudados, para o controle biológico clássico do minador. Esta espécie tem sido utilizada em larga escala no controle de $P$. citrella no Brasil e no exterior (PAIVA et al., 2000; LinARES et al., 2001).

Do material coletado nas vinte e seis ocasiões de amostragem realizadas no primeiro ano de estudos, foram obtidos 101 parasitóides nos dois pomares.

No pomar de 'Montenegrina', foram identificadas seis espécies de parasitóides emergidos de pupas de $P$. citrella. Cirrospilus neotropicus apareceu em maior freqüência (41,5\%), seguida de Elasmus sp. 1 (30,8\%) e C. floridensis (12,2\%) (Tab. I). Nesta área, as espécies de Cirrospilus apresentaram dominância, somando 53,7\% do total registrado. Ageniaspis citricola, exótica, não foi registrada no primeiro ano em 'Montenegrina'.

Neste mesmo ano, na área correspondente a 'Murcott', foram identificadas também seis espécies de parasitóides (Tab. I), sendo A. citricola a mais freqüente (45\%). Cirrospilus neotropicus e C. floridensis, somadas, representaram 33,4\% e Elasmus sp. 1, 18,3\% do total de parasitóides encontrados.

Embora a maioria das espécies esteja presente nas duas áreas, observam-se diferenças na estrutura do complexo de parasitóides ocorrentes, por exemplo, Sympiesis sp. foi encontrada exclusivamente na área de 
Tabela I. Parasitóides de Phyllocnistis citrella registrados, freqüência relativa e constância das espécies em dois pomares de citros em Montenegro (29 $68^{\prime}$ S, 51 $\left.{ }^{\circ} 46^{\prime} \mathrm{W}\right)$, RS. (Julho de 2001 a junho de 2002 e julho de 2002 a junho de 2003 ).

\begin{tabular}{|c|c|c|c|c|c|c|c|c|c|c|c|c|}
\hline \multirow[b]{3}{*}{ Espécies } & \multicolumn{7}{|c|}{ 'Montenegrina' } & \multicolumn{5}{|c|}{ 'Murcott' } \\
\hline & \multicolumn{4}{|c|}{$\begin{array}{c}\text { Ano } 1 \\
2001-2002\end{array}$} & \multicolumn{2}{|c|}{$\begin{array}{c}\text { Ano } 2 \\
2002-2003\end{array}$} & \multicolumn{4}{|c|}{$\begin{array}{c}\text { Ano } 1 \\
2001-2002\end{array}$} & \multicolumn{2}{|c|}{$\begin{array}{c}\text { Ano } 2 \\
2002-2003\end{array}$} \\
\hline & $\mathrm{n}$ & $\begin{array}{l}\text { Freqüência } \\
\text { relativa \% }\end{array}$ & $\begin{array}{c}\text { Constância } \\
\%\end{array}$ & $\mathrm{n}$ & $\begin{array}{c}\text { Freqüência } \\
\text { relativa \% }\end{array}$ & $\begin{array}{c}\text { Constância } \\
\%\end{array}$ & $\mathrm{n}$ & $\begin{array}{l}\text { Freqüuência } \\
\text { relativa \% }\end{array}$ & $\begin{array}{c}\text { Constância } \\
\%\end{array}$ & $\mathrm{n}$ & $\begin{array}{l}\text { Freqüência } \\
\text { relativa } \%\end{array}$ & $\begin{array}{c}\text { Constância } \\
\%\end{array}$ \\
\hline Elasmus sp.1 & 14 & 30,8 & 23,1 & 2 & 0,4 & 23,1 & 11 & 18,3 & 19,2 & 6 & 3,9 & 11,5 \\
\hline Elasmus sp. 2 & 1 & 2,4 & 3,8 & 9 & 2 & 7,7 & 1 & 1,7 & 3,8 & 2 & 1,3 & 7,7 \\
\hline Cirrospilus neotropicus & 17 & 41,5 & 26,9 & 53 & 11,6 & 30,8 & 13 & 21,7 & 26,9 & 13 & 8,6 & 19,2 \\
\hline Cirrospilus floridensis & 5 & 12,2 & 19,2 & 15 & 3,3 & 15,4 & 7 & 11,7 & 11,5 & 4 & 2,6 & 11,5 \\
\hline Sympiesis sp. & 2 & 4,9 & 7,7 & 0 & 0 & 0 & 0 & 0 & 0 & 0 & 0 & 0 \\
\hline Galeopsomyia fausta & 2 & 4,9 & 3,8 & 7 & 1,5 & 11,5 & 1 & 1,6 & 3,8 & 6 & 3,9 & 7,7 \\
\hline Ageniaspis citricola & 0 & 0 & 0 & 371 & 81,2 & 34,6 & 27 & 45 & 23,1 & 121 & 79,6 & 26,9 \\
\hline
\end{tabular}

'Montenegrina'; na freqüência das espécies, Elasmus sp.1 ocorre com maior frequiência em 'Montenegrina' $(30,8 \%)$ do que em 'Murcott' $(18,3 \%)$ e quanto à dominância das mesmas, em 'Murcott', A. citricola foi dominante.

A presença de A. citricola, no primeiro ano, na área cultivada com 'Murcott', deve-se possivelmente à maior proximidade desta com o local onde ocorreu a liberação do microhimenóptero para o controle de $P$. citrella.

No segundo ano foi amostrado um total de 609 parasitóides. Observa-se que, embora o número de indivíduos tenha sido maior, o de espécies não aumentou, indicando que, provavelmente, as obtidas no primeiro ano, representam, de fato, o complexo principal de parasitóides associados a $P$. citrella no local de estudo. O número cumulativo de espécies ao longo das amostragens estabilizou-se, o que fica claro nas curvas de suficiência amostral (Fig. 1) indicando que o número de espécies registradas reflete o número presente nas áreas.

Em 'Montenegrina', no segundo ano, não houve o registro de Sympiesis sp. As duas espécies de Cirrospilus, que, no ano anterior compunham 53,7\% dos registros, neste ano, juntas, somaram somente $14,9 \%$ (Tab. I).

$\mathrm{Na}$ área de 'Murcott', A. citricola aumentou a freqüência de $45 \%$ no primeiro ano para $79,6 \%$ no segundo. Embora não se tenha constatado o desaparecimento de nenhuma espécie, as do gênero Cirrospilus baixaram sua freqüência de $33,4 \%$ para 11 , $2 \%$ (Tab. I).

Em ambas áreas houve uma grande modificação na estrutura do complexo de parasitóides, alterandose a presença, a freqüência e a constância das espécies (Tab. I).

Percebe-se nitidamente um aumento na população de $A$. citricola em ambas as áreas (Tab. I), o que aponta a rápida ocupação do espaço por este parasitóide exótico. Na área de 'Montenegrina', onde, no primeiro ano de amostragem, A. citricola não foi registrada, a mesma aparece, no segundo, como a espécie mais freqüente. Este dado reforça a idéia que A. citricola está se dispersando a partir do seu ponto de liberação, próximo à área de 'Murcott' e colonizando outras áreas.

Por outro lado, observa-se uma diminuição na freqüência dos parasitóides autóctones e um aumento na da espécie exótica. Este fato pode estar relacionado ao comportamento especialista e poliembriônico de $A$. citricola. Essas características, aliadas ao fato dessa espécie ter sido introduzida e estar, num primeiro momento, livre de inimigos naturais, pode, em parte, explicar sua maior freqüência. Da mesma forma, estes dados podem estar indicando uma tendência de exclusão competitiva. Conforme Ricklefs (1996), quando várias espécies de parasitóides são introduzidas simultaneamente para controlar populações de pragas na mesma localidade e para explorar o mesmo recurso, é de se esperar que exclusões competitivas ocorram sob estas condições, com o deslocamento das espécies pré-existentes para outros nichos ou, mesmo, a extinção local de uma população. No presente caso, apenas uma espécie exótica $(A$. citricola) foi introduzida e as observações dos anos sucessivos (Tab. I) sugerem que a mesma provocou uma mudança no conjunto de espécies de parasitóides associados a $P$. citrella nas áreas estudadas. Entretanto, o período de dois anos representa pouco tempo de observação. Somente uma continuidade das amostragens nas mesmas áreas poderia apontar se esta mudança representa apenas um deslocamento temporário ou se acarretará a extinção de populações locais.

SÁ et al. (1999) também observaram uma mudança na estrutura do complexo de parasitóides após o estabelecimento de $A$. citricola em pomares de citros, em Jaguariúna, SP. Os autores verificaram que, antes da introdução do parasitóide exótico, G. fausta predominava com cerca de $92 \%$ de freqüência, diminuindo, após, para 38\%. Da mesma forma, espécies menos frequientes deixaram de ser constatadas.

Os registros de constância no presente estudo revelam que, nos dois pomares, no primeiro ano, houve uma grande variação das espécies presentes nas diferentes ocasiões de amostragem, não evidenciando uma espécie mais constante. Já no segundo ano, $A$. citricola mostrou-se constante na área de 'Montenegrina', estando presente em cerca de 35\% das amostragens realizadas.

Cirrospilus neotropicus, C. floridensis e Elasmus sp. 1 embora em menor frequiência, também estiveram presentes em grande parte das ocasiões de amostragem no segundo ano (Tab. I). Isto pode indicar 

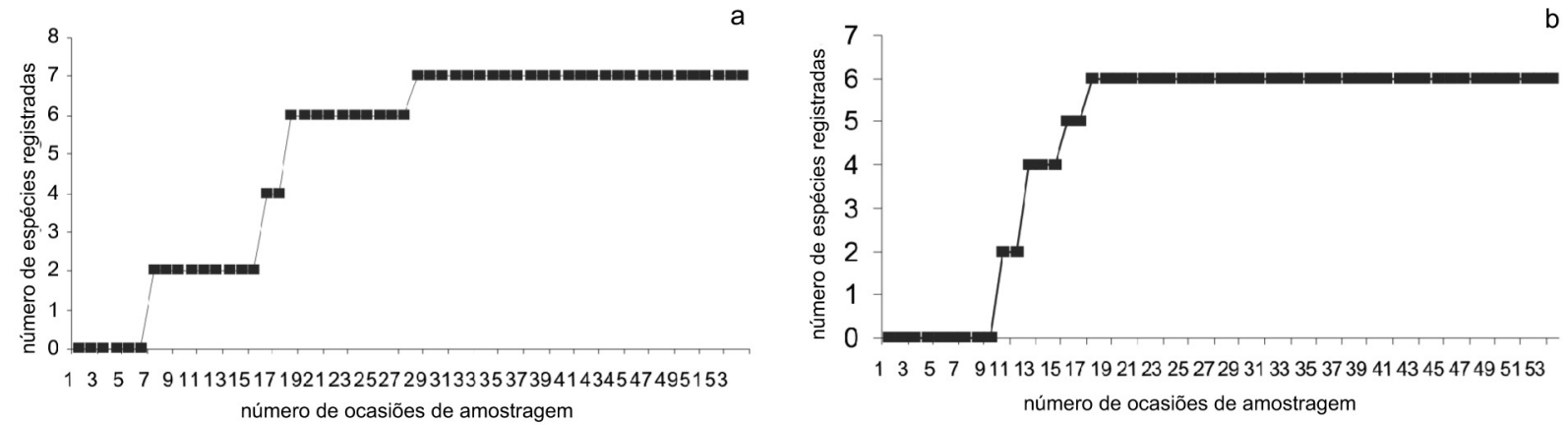

Fig. 1. Número cumulativo de espécies de parasitóides de Phyllocnistis citrella obtidas em sucessivas amostragens nos pomares de (a) 'Montenegrina' e (b) 'Murcott' em Montenegro, RS (29 68'S, 51² 46'W), RS, de julho de 2001 a junho de 2003.

que as espécies estejam estabelecendo-se, com segregação parcial de nichos, visto que A. citricola é um endoparasitóide de ovos e do primeiro instar de $P$. citrella, enquanto que as outras espécies caracterizamse por serem ectoparasitóides do quarto instar e de pré-pupas (Argov \& Rössler, 1996). Para Browing et al. (1996), os nichos ocupados por Eulophidae variam de minas larvais a celas de pré-pupas ou pupas. Neste caso, a competição maior entre as espécies ocorre entre os ínstares tardios e pré-pupas, onde cada instar serve a duas ou mais espécies nativas. Entretanto, BRowING et al. (1996), em estudo realizado em pomares de citros na Flórida, registraram que o impacto de parasitóides nativos cresceu desde 1993, quando iniciou o monitoramento, e que mais espécies foram recrutadas no complexo.

No presente estudo, observou-se que, com a introdução de $A$. citricola nas áreas adjacentes aos pomares e seu posterior estabelecimento nas áreas de 'Murcott' e 'Montenegrina', houve uma redução no número de espécies de parasitóides e na dominância das mesmas. Segundo HowARTH (1991) em casos extremos, a introdução de espécies exóticas para controle de pragas pode causar tanto um drástico declínio nas populações de espécies comuns, quanto levar as raras, próximo à extinção local. Neste caso, ocorre uma diminuição na quantidade e diversidade de inimigos naturais presentes na área de cultivo.

As pesquisas sobre sistemas múltiplos de cultivo enfatizam a grande importância da diversidade em um cenário agrícola, com o propósito de aumentar a estabilidade destes (Altieri, 1989). Neste sentido é que, para Michaud (2002), o controle biológico em cultivos perenes deve estar baseado em um complexo de inimigos naturais endógenos, cujo conhecimento das espécies, da biologia e das interações, é fundamental para que o manejo de pragas seja bem sucedido.

Os registros da ocorrência e a avaliação da real contribuição de parasitóides nativos e de predadores associados à redução populacional de $P$. citrella oportunizam uma estimativa realista do efeito do estabelecimento de inimigos naturais exóticos e podem apontar a necessidade do desenvolvimento e da implementação de outras estratégias de manejo, visando equilibrar o agroecossistema, minimizar custos e aumentar a produtividade local.
Agradecimentos. Ao Dr. Valmir Antônio Costa, do Instituto Biológico de Campinas, pelas identificações e ao Dr. John La Salle, CSIRO Entomology (Austrália), pela identificação de $C$. floridensis. À CAPES e ao CNPq pela concessão das bolsas de pesquisa ao primeiro e segundo autor, respectivamente.

\section{REFERÊNCIAS BIBLIOGRÁFICAS}

Altieri, M. A. 1989. Agroecologia: as bases científicas da agricultura alternativa. PTA/FASE. 240p.

Argov, Y. \& Rössler, Y. 1996. Introduction, release and recovery of several exotic natural enemies for biological control of the citrus leafminer Phyllocnistis citrella, in Israel. Phytoparasitica 24:33-38.

Becker, R. F. P. \& Moraes, L. A. H. 2001. Relatório do programa de melhoria da fruta cítrica do vale dos rios Caí e Taquari. Taquari, Fepagro. 24p.

Browning, H. W.; Peña, J. E. \& Stansly, P. A. 1996. Evaluating impact of indigenous parasitoids on population of citrus leafminer. Folia Entomologica Mexicana 79:14-15.

Burks, R. A. 2001. Key to the Neartic genera of Eulophidae, subfamilies Entedoninae, Euderinae and Eulophinae/ Hymenoptera: Chalcidoidea). Disponível em: <http://cache.ucr.edu/\%7Eheraty/Eulophidae>. Acesso em: 05.2003

Callegari-Jacques, S. M. 2004. Bioestatística: princípios e aplicações. Porto Alegre, Artmed. 255p.

Chagas, M. C. M. \& Parra, J. R. P. 2000. Phyllocnistis citrella Stainton (Lepidoptera: Gracillariidae): técnica de criação e biologia em diferentes temperaturas. Anais da Sociedade Entomológica do Brasil 29:227-235.

Dajoz, R. 1973. Ecologia Geral. Petrópolis, Vozes. 472p.

Garcia, R. R. M.; Carabagialle, M. C.; Sá, N. L. A. \& Campos, J. V. 2001. Parasitismo natural de Phyllocnistis citrella Stainton, 1856 (Lepidoptera, Gracillariidae, Phyllocnistinae) no oeste de Santa Catarina, Brasil. Revista Brasileira de Entomologia 45:139-143.

Generalitat Valenciana. 1996. El minador de las hojas de los cítricos (Phyllocnistis citrella St.). Valencia, Conselleria de Agricultura Y Medio Ambiente. 8p.

GravenA, S. 1998. Lagarta minadora dos citros no Brasil. Laranja 17:286-288.

Jahnke, S. M.; Redaelli, L. R. \& Diefenbach, L. M. G. 2005. Primeiro registro de Cirrospilus floridensis Evans 1999, parasitóide de Phyllocnistis citrella Stainton no Brasil. Ciência Rural 35(2):459-461

Heppner, J. B. 1993. Citrus leafminer, Phyllocnistis citrella (Lepidoptera: Gracillariidae: Phyllocnistinae) in Florida. Tropical Lepidoptera 4:49-64.

Howarth, F. G. 1991. Environmental impacts of classical biological control. Annual Review of Entomology 36:485-509.

Linares, B.; Hernández, J.; Morillho, J. \& Hernández, L. 2001. Introducción de Ageniaspis citricola Logvinoskaya, 1983 (Hymenoptera: Encyrtidae) para control de Phyllocnistis citrella Stainton (Lepidoptera: Gracillariidae) en el estado 
Yaracuy, Venezuela. Entomotropica 16:143-145.

Michaud, J. P. 2002. Classical biological control: a critical review of recent programs against citrus pests in Florida. Annals of the Entomological Society of America 94:531-540.

Montes, S. M. N. M.; Boliani, A. C.; Papa, G.; Cerávolo, L. C.; Rossi, A. C. \& Namekata, T. 2001. Ocorrência de parasitóides da larva minadora dos citros, Phyllocnistis citrella Stainton, no município de Presidente Prudente, SP. Arquivos do Instituto Biológico de São Paulo 68:63-66.

Nascimento, F. N.; Santos, W. da S.; Pinto, J. DE M. \& Cassino, P. C. R. 2000. Parasitismo em larvas de Phyllocnistis citrella Stainton (Lepidoptera: Gracillariidae) no estado do Rio de Janeiro. Anais da Sociedade Entomológica do Brasil 29:377-379.

Paiva, P. E. B.; Gravena, S. \& Amorin, L. C. S. 2000. Introdução do parasitóide Ageniaspis citricola Logvinovskaya para controle biológico da minadora das folhas dos citros Phyllocnistis citrella Stainton no Brasil. Anais da Sociedade Entomológica do Brasil 29:149-154.

Penteado-Dias, A.; Gravena, S. A.; Paiva, P. E. \& Pinto, R. 1997. Parasitóides de Phyllocnistis citrella Stainton (Lep.: Gracillariidae) no estado de São Paulo. Laranja 18:79-84.

Prates, H. S.; Nakano, O. \& Gravena, S. A. 1996. A "minadora das folhas de citros" Phyllocnistis citrella Stainton, 1856. Campinas, CATI. 3p. (Comunicado Técnico, 129).
Ricklefs, R. E. 1996. A economia da natureza. 3. ed. Rio de Janeiro, Guanabara Koogan. 470p.

Sá, L. A. N.; Costa, V. A.; Oliveira, W. P. \& Almeida, G. R. 2000. Parasitoids of Phyllocnistis citrella in Jaguariúna, State of São Paulo, Brazil, before and after the introduction of Ageniaspis citricola. Scientia Agrícola 57(4):799801.

SÁ, L. A. N.; Costa, V. A.; Tambasco, F.; Oliveira,W. \& Almeida, G. 1999. Parasitóides da larva minadora da folha dos citrus, Phyllocnistis citrella Stainton, estudos no laboratório de quarentena "Costa Lima" em Jaguariúna, SP. Jaguariúna, Embrapa Meio Ambiente. p.1-4. (Comunicado Técnico, 2).

Schauf, M. E.; Lasalle, J. \& Wijesakara, G. A. 1998. The Genera of Chalcid parasitoids (Hymenoptera: Chalcidoidea) of citrus leafminer Phyllocnistis citrella Stainton (Lepidoptera: Gracillariidae). Journal of Natural History 32:1001-1056.

Urbaneja, A.; Jacas, J.; Verdú, M. J. \& Garrido, A. 1998. Dinamica e impacto de los parasitoides autoctonos de Phyllocnistis citrella Stainton, en la comunidad valenciana. Investigacion Agraria: Produccion Y Proteccion Vegetales 13:409-423.

Willink, E.; Salas, H. \& Costilla, M. A. 1996. El minador de la hoja de los cítricos, Phyllocnistis citrella en el NOA. Avance Agroindustrial 16:15-20 